Research Article

\title{
Prevalence of Anemia and Undernutrition of Adolescent Females in Selected Schools in Ghana
}

\author{
Marina Aferiba Tandoh, ${ }^{1}$ Abigail Owusuaa Appiah $\mathbb{D}^{1},{ }^{1}$ and Anthony Kwaku Edusei ${ }^{2}$ \\ ${ }^{1}$ Department of Biochemistry and Biotechnology, College of Science, Kwame Nkrumah University of Science and Technology, \\ $P M B$, Kumasi, Ghana \\ ${ }^{2}$ Department of Health Promotion and Disability Studies, School of Public Health, \\ Kwame Nkrumah University of Science and Technology, Kumasi, Ghana \\ Correspondence should be addressed to Abigail Owusuaa Appiah; maamewusuakyeaw@gmail.com
}

Received 7 October 2020; Revised 28 November 2020; Accepted 26 December 2020; Published 9 January 2021

Academic Editor: Mohammed S. Razzaque

Copyright ( 2021 Marina Aferiba Tandoh et al. This is an open access article distributed under the Creative Commons Attribution License, which permits unrestricted use, distribution, and reproduction in any medium, provided the original work is properly cited.

\begin{abstract}
Anemia among adolescent females is a major worldwide public health problem which should be given appropriate attention. Half of all anemic cases are caused by iron deficiency. In addition to anemia, poor nutrition is also a challenge in sub-Saharan Africa. This study determined the prevalence of anemia and undernutrition among adolescent females in school. A cross-sectional study was conducted among 151 adolescent females in four basic schools in the Ahafo region of Ghana. The hemoglobin level and anthropometry measures of participants were taken to determine their anemic and nutritional status. The prevalence of anemia among adolescent females in school was $50.3 \%$. Out of the 151 participants, $6.6 \%, 19.9 \%$, and $23.8 \%$ were severely anemic, moderately anemic, or mildly anemic, respectively. Only $2 \%$ of the participants were underweight, but the rate of stunting was 26.5\%. The notable high prevalence of anemia that was found among adolescent females was correlated with other health and wellness concerns. Anemia and under nutrition negatively affect academic performance, productivity, and general wellbeing of adolescents; therefore, effective measures should be put in place to correct and eradicate these nutritional problems.
\end{abstract}

\section{Introduction}

Adolescence is a transition period between childhood and adulthood [1]. During adolescence, there are periods of rapid mental, physical, psychological, and cognitive development [1]. Optimum cognitive development enhances school performances, problem solving abilities, and productivity of adolescents [1]. These developmental changes influence the nutritional needs of adolescents [1]. Nutritional status is defined as an individual's health condition as it is influenced by the intake and utilization of nutrients [2]. Nutritional status can be determined through anthropometric measurement of the body composition, biochemical measurement of serum protein, micronutrients and metabolic parameters, and assessment of altered nutritional requirement and social issues that may affect adequate nutrient intake [2]. Suboptimal nutritional status including excess and inadequate caloric intake and micronutrient deficiencies could directly affect physical, psychological, and physiological functioning of the body [2]. Nutritional status can therefore be used as a determinant of growth, health, risk of chronic diseases, and long-term quality of life [1]. Adequate nutrition in adolescents serves as an investment into their adult health [3]. It also prevents the vicious cycle of malnutrition, chronic diseases, and poverty [3].

Worldwide, nutrient deficiencies are considered a dominant cause of anemia, and in sub-Saharan Africa, it is the major cause of anemia in adolescent females [4]. Ghana is among the countries with the highest burden of under nutrition, iron deficiency anemia, low serum retinol, and stunting [5]. Ghana was ranked 135 out of 187 countries with highest burden of malnutrition in the world [6].

The prevalence of nutritional anemia is estimated to be $40 \%$ worldwide [7]. Nutritional anemia may be defined as a low hemoglobin concentration due to deficiencies in hemopoietic nutrients such as iron, folate, and vitamin $B_{12}[8]$. 
Deficiencies in hematinic nutrients are not only the most common causes of anemia but also are the easiest to treat once the specific deficient nutrient is determined [9]. Inadequate consumption of hemopoietic nutrients such as iron, vitamin $\mathrm{B}_{12}$, and folate is a major cause of low hemoglobin concentration in the blood [8]. Low bioavailability of iron and other hematinic nutrients could be a contributory factor to anemia [9].

In preventing anemia, dietary adequacy and diversity are critical. However, most adolescents do not consider the nutritional composition of their meals but rather think about satisfying their hunger [10]. The increase rate of nutritional problems such as anemia and being overweight or obese in adolescents could be associated with high intake of energy-dense foods with little or no essential micronutrients [11].

Unfortunately, anemia can be caused by other factors, which are not associated with dietary intake. These causes include worm infestation, malaria, chronic blood loss, gastrointestinal bleeding, cancers, vascular lesions, and ulcers [8].

Anemia has a negative effect on the overall growth, cognitive development, and academic performance of adolescents [12]. Anemia can also result in fatigue and low productivity, which have a negative impact on the economy. Anemic adolescents who get pregnant also have an increased risk of morbidity, mortality, and poor birth outcomes such as low birth weight, premature birth, and still birth [12].

The health of adolescents has not been a major concern, and consequently, there has been limited research in the area of adolescent nutrition particularly in developing countries, hence the reason for this study. This study was conducted to determine the prevalence of stunting, underweight, and anemia among adolescent females in school in Ghana. The prevalence of anemia in this study was $50.3 \%$ which is an indication that anemia is still a public health problem in Ghana. The findings will therefore be relevant in the development of effective interventions to reduce anemia in Ghana.

\section{Materials and Methods}

2.1. Study Design and Setting. A cross-sectional study was conducted among adolescent females aged 10-19 years. Participants were recruited from four government-owned schools in the Tano North municipal district of the Ahafo region, Ghana.

2.2. Study Participants and Sample Size. Adolescent females attending upper primary and junior high schools were included in the study. A sample size of 260 was determined using Cochran's formula [13]. Out of about 280 female adolescents in the four basic schools, parents of 151 participants consented for their wards to participate in the study. A written assent was obtained from all the participants before data collection, since they were all below 18 years.
2.3. Data Collection. A structured questionnaire was used to obtain information from participants. Data collected include sociodemographics, anthropometry (weight, height, and body mass index), and biochemical data (hemoglobin level).

2.4. Anthropometric Measurements. Weight was measured with the Omron scale (Omron BF511) to the nearest 0.1 kilogram. The height of the participants was measured with a Seca stadiometer to the nearest 0.1 centimeter. The body mass index (BMI) was determined using the weight and height of the participants by calculating the weight $(\mathrm{kg}) /$ height $\left(\mathrm{m}^{2}\right)$. Participants were classified as being underweight, normal, overweight, or obese with the use of the WHO BMI for age chart. Stunting among the adolescent females was determined with the WHO height for age chart [14].

2.5. Hemoglobin. Hemoglobin (Hb) levels of all participants were determined using a portable hemoglobin meter URIT 12 manufactured by Medical Electronic Group Company Limited in China. A drop of the capillary blood was obtained from the middle finger after a prick and was placed unto the hemoglobin meter strip. Based on the WHO classification, $\mathrm{Hb}$ levels below $8 \mathrm{~g} / \mathrm{dL}$ were classified as severe anemia, $8-10.99 \mathrm{~g} / \mathrm{dL}$ as moderate anemia, and $11-11.9 \mathrm{~g} / \mathrm{dL}$ as mildly anemic, and $\mathrm{Hb}$ of $12 \mathrm{~g} / \mathrm{dL}$ and above was classified as normal [15].

2.6. Data Analysis. Statistical Package for Social Sciences (SPSS) version 26.0 was used to determine the frequency distribution of participants who were anemic, stunted, underweight, and obese. Nutritional status of participants was determined with the WHO's recommended age-specific cutoff points of BMI and height. Tables and figures were used to summarize the results obtained. Continuous variables with normal distribution were expressed as mean $\pm \mathrm{SD}$ (standard deviation), while proportions were calculated for discontinuous ones.

\section{Results}

The participants were classified into early, middle, and late adolescents based on their ages. The ages ranged from 10 to 17 years. The majority of the participants were in early adolescence. Majority of the mothers (61.4\%) and fathers (44.6\%) of the participants were traders and farmers, respectively. Also, $19.3 \%$ of mothers and $14.5 \%$ of fathers of participants had no formal education. Table 1 presents the above characteristics of the participants.

To determine the mean hemoglobin level of participants, the prevalence of anemia and anemia classification among the adolescent females is displayed in Table 2, and descriptive statistics was performed. More than half $(50.3 \%)$ of the adolescent females were anemic. Out of the 76 anemic participants, $47.4 \%$ were mildly anemic, $39.5 \%$ were moderately anemic, and $13.1 \%$ were severely anemic. 
TABLE 1: Characteristics of participants.

\begin{tabular}{lc}
\hline Variable & Mean \pm SD \\
& $N=151$ \\
\hline Age in years & $12.87 \pm 1.89$ \\
\hline Variable & Frequency $(\%)$ \\
Age distribution of participants & \\
Early adolescence (10-13 yrs) & $106(62.7)$ \\
Middle adolescence (14-16 yrs) & $43(34.9)$ \\
Late adolescence (17-19yrs) & $2(2.4)$ \\
Education of mother & \\
No formal education & $25(19.3)$ \\
Basic (JHS) & $65(47)$ \\
Secondary (SHS) & $48(26.5)$ \\
Tertiary & $13(7.2)$ \\
Education of father & \\
No formal education & $24(14.5)$ \\
Basic (JHS) & $38(26.5)$ \\
Secondary (SHS) & $59(42.1)$ \\
Tertiary & $30(16.9)$ \\
Occupation of mother & \\
Trading & $97(61.4)$ \\
Farming & $34(25.3)$ \\
Others & $20(13.3)$ \\
Occupation of father & \\
Trading & $15(13.3)$ \\
Farming & $60(44.6)$ \\
Driving & $24(19.3)$ \\
Teaching & $10(4.8)$ \\
Mason & $8(1.2)$ \\
Others & \\
\hline
\end{tabular}

Others ${ }^{1}$, teaching, hairdressing, cleaning, police officer, and cooks. Others ${ }^{2}$, carpentry, barbering, teaching, trading, banking, pastor, and doctor. SD, standard deviation.

TABle 2: Prevalence and classification of anemia among adolescent females.

\begin{tabular}{lc}
\hline Variable & $\begin{array}{c}\text { Mean } \pm \text { SD } \\
N=151\end{array}$ \\
\hline Hb levels & $11.6 \pm 2.1$ \\
Prevalence of anemia (frequency (\%)) & $76(50.3)$ \\
$\quad$ Anemic & $75(49.7)$ \\
$\quad$ Not anemic & \\
Anemia classification (frequency (\%)) & $10(6.6)$ \\
$\quad$ Severe anemia & $30(19.9)$ \\
Moderate anemia & $36(23.8)$ \\
$\quad$ Mild anemia &
\end{tabular}

The anthropometric measures showed that majority $(82.1 \%)$ of the participants had a normal body mass index (BMI). Two (1.3\%) participants were classified as obese, and $2 \%$ was classified as underweight. Figure 1 shows the BMI classification of the participants. Table 3 presents the mean BMI and height for age of the participants. Stunting among the adolescent females was $26.5 \%$.

Table 4 presents the prevalence of anemia and the presence of malaria. Majority (72.4\%) of the anemic participants had normal BMI with few of them being obese (2.6\%) and underweight (1.3\%). About $60.5 \%$ of the anemic participants reported to have had malaria prior to the study. The prevalence of anemia and the presence of malaria are displayed in Table 5.

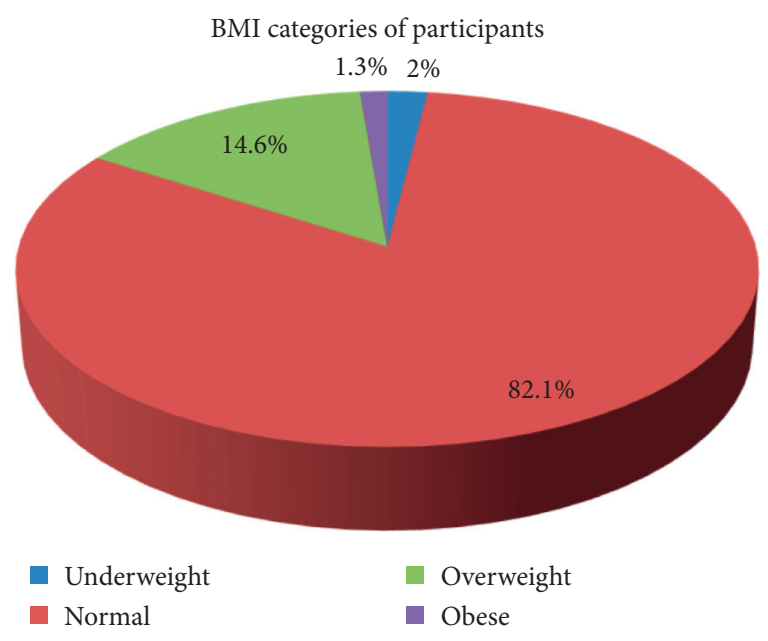

FIgURE 1: BMI classification of participants.

TABle 3: Anthropometric measures of adolescent females.

\begin{tabular}{lc}
\hline Variable & Mean \pm SD \\
$N=151$ \\
\hline Weight $(\mathrm{kg})$ & $42.3 \pm 10.2$ \\
Height $(\mathrm{cm})$ & $148.6 \pm 14.1$ \\
BMI $\left(\mathrm{kg} / \mathrm{m}^{2}\right)$ & $18.7 \pm 2.9$ \\
Height for age (frequency $(\%))$ & \\
Stunted & $40(26.5)$ \\
Not stunted & $111(73.5)$ \\
\hline
\end{tabular}

\section{Discussion}

The overall mean age of participants was $12.3 \pm 1.76$ years which ranged from 10 to 17 years. Most of the participants $(70.2 \%)$ were within the ages of 10-13 years (early adolescence) and few of them (1.3\%) were in their late adolescence (17-19 years). This was similar to a study conducted among adolescent females in Bangladesh where most (50\%) of the participants were in their early adolescence, $30.7 \%$ in middle adolescence, and $19.3 \%$ in late adolescence [16]. In this study, a majority of the participants were in their early adolescence which could be attributed to the typical demographics of most adolescents in Ghana upper primary and junior high schools.

The majority of participants had normal BMIs with few of them being underweight, overweight, and obese. A different study conducted in Bangladesh showed similar results where $80 \%$ of adolescent females had a normal BMI, with few of them being thin or severely thin and overweight [16]. Out of the 124 participants with normal BMI, $44.4 \%$ were anemic. The findings of the study showed that $81.8 \%$, $50 \%$, and $50 \%$ of the overweight, underweight, and stunted participants, respectively, were anemic. The two participants who were obese were also anemic. The findings of this study are indications that adolescent females could be anemic regardless the BMI class they belong. However, the results of the study showed that greater percentages of overweight and obese participants were anemic and that may not be a good reflection since the obese and overweight participants were few. 
TABle 4: Prevalence of anemia and BMI classification.

\begin{tabular}{lcc}
\hline BMI classification & Anemic (frequency (\%)) & Not anemic (frequency (\%)) \\
$N=75$
\end{tabular}

Table 5: Prevalence of anemia and the presence of malaria.

\begin{tabular}{lcc}
\hline Variable & Anemic (frequency (\%)) & Not anemic (frequency (\%)) \\
& $N=76$ & $N=75$ \\
\hline Presence of malaria & $46(60.5)$ & $29(38.7)$ \\
No malaria & $30(39.5)$ & $46(61.3)$ \\
\hline
\end{tabular}

The prevalence of anemia among adolescent females in school was found to be $50.3 \%$ in this study. The high prevalence of anemia in this study is similar to a study conducted in India where $64.7 \%$ of adolescent females were found to be anemic [17]. The prevalence of anemia among female adolescents in this study is high compared to that of a study conducted in Ethiopia where the prevalence ranged from $9.3 \%$ to $34.8 \%$ in female adolescents [18]. The prevalence of anemia was also found to be $21.1 \%$ in school-going girls in western Kenya, and it is also lower compared to the findings of this study [4]. The differences in the anemia prevalence could be due to variation in the geographical area and study participants. In Ghana, the prevalence of anemia in women of reproductive age (15-49 years) was $42.4 \%$ in 2014 [19], and the increase in anemia prevalence in this study could be attributed to the age differences. In the present study, the prevalence of severe anemia was $6.6 \%$, while moderate and mild anemia were $19.9 \%$ and $23.8 \%$, respectively, and it is in line with a different study where the prevalence of severe, moderate, and mild anemia was $0.2 \%, 6.3 \%$, and $33.0 \%$, respectively [20].

The high prevalence of anemia in adolescence could be associated with the rapid growth of the adolescents experience during this period which increases their requirement for hematinic nutrients such as iron, folate, and vitamin $B_{12}$. A study conducted in Ghana showed that animal foods per calorie have a higher influence on hemoglobin levels [21]. However, a typical Ghanaian diet is chiefly carbohydrate with little animal protein.

The onset of menarche, loss of blood through menstruation, and malaria could also be contributory factors to the high prevalence of anemia in adolescent females [1]. Majority of the anemic participants (60.5\%) reported to have been diagnosed and treated for malaria one month prior to the study, but since it was self-reported, it may not be a true reflection. Sensitive laboratory indicators such as serum ferritin, serum iron, and full blood count would have been very useful, but it was not performed as it would drive the cost of the study high.
In December 2018, the Ministry of Health together with the Ghana Education Service and other agencies started the first phase of the intermittent weekly girl iron folic acid tablet supplementation (GIFTS) program in Bono, Ahafo, Northern, upper east, and Volta regions of Ghana to address the high prevalence of anemia (26.4\%) among adolescent girls of 15-19 years [22]. The evaluation of the GIFTS program revealed that majority of adolescent females missed taking the iron folic acid (IFA) tablets because the tablets were not received from their teachers. Some adolescent females also perceived the IFA tablet as either a contraceptive or fertility drug and therefore refused to take the tablets [23]. The findings of this study showed that the prevalence of anemia in adolescent females is still high in Ghana, even though there have been an intervention in the form of school-based IFA supplementation $(60 \mathrm{mg}$ elemental iron and $400 \mu \mathrm{g}$ of folic acid), an intervention reported by other studies to be feasible and effective in preventing anemia [24].

\section{Conclusion and Recommendations}

The prevalence of anemia among adolescent females in school is still a public health problem in Ghana. Effective nutritional interventions in the form of food fortification and supplementation should be provided to prevent anemia and its effects on academic performance, productivity, and overall growth of adolescent females. The girl iron folic acid tablet supplementation programme should be made effective through sensitization, expanded training, and streamlined monitoring in all basic schools. Majority of the participants had normal BMI with few of them being underweight, overweight, or obese. Nutrition education in schools could help eradicate undernutrition and stunting among adolescent females.

\section{Data Availability}

The primary data used to support the findings of this study are available from the corresponding author upon request. 


\section{Conflicts of Interest}

The authors declare that they have no conflicts of interest.

\section{References}

[1] M. Massey-stokes, "Adolescent nutrition: needs and recommendations for practice," The Clearing House: A Journal of Educational Strategies, Issues and Ideas, vol. 75, no. 6, p. 286, 2002.

[2] FAO, Nutritional Status Assessment and Analysis: Nutritional Status and Food Security, Food and Agriculture Organization, Rome, Italy, 2007.

[3] WHO, Nutrition in Adolescence-Issues and Challenges for the Health Sector, World Health Organization, Geneva, Switzerland, 2005.

[4] D. Nelima, "Prevalence and determinants of anaemia among adolescent girls in secondary schools in yala division Siaya district, Kenya," Universal Journal of Food and Nutrition Science, vol. 3, no. 1, pp. 1-9, 2015.

[5] J. Ayamba and L. Boateng, Relation Ship between Dietary Diversity and Haemoglobin Concentration of Women in Reproductive Age in Three Communities in the Binduri District of the Upper East Region of Ghana, University of Ghana Space, Accra, Ghana, 2018, http://ugspace.ug.edu.gh.

[6] National Nutritional Policy for Ghana 2013-1017, 2013.

[7] D. Karva, S. Agarkhedkar, and S. Deshpande, "Prevalence of anemia in adolescent girls and its co-relation with demographic factors," International Journal of Health Science, vol. 3, no. 4, pp. 3-7, 2013.

[8] H. A. Alaliwi, G. A. Abukashba, H. M. Alhussain et al., "Nutritional anemia types and management," The Egyptian Journal of Hospital Medicine, vol. 70, no. 6, pp. 906-911, 2018.

[9] O. E. Ifeanyi, “A review on nutritional anaemia," International Journal of Advanced Multidisciplinary Research, vol. 5, no. 4, pp. 11-15, 2018.

[10] C. N. A. Buxton, "Ghanaian junior high school adolescents dietary practices and food preferences: implications for public health concern," Journal of Nutrition and Food Sciences, vol. 4, no. 5, pp. 1-9, 2014.

[11] C. M. Klutse, "An investigation into the adolescents nutritional and dietary requirements among secondary schools in the volta region of Ghana," Global Research Journal of Science and Nature, vol. 1, no. 1, pp. 1-5, 2015.

[12] G. Mengistu, M. Azage, and H. Gutema, "Iron deficiency anemia among in-school adolescent girls in rural area of Bahir Dar city administration, North West Ethiopia," Anemia, vol. 2019, Article ID 1097547, 9 pages, 2019.

[13] W. G. Cochran, Sampling Techniques, John Wiley \& Sons, New York, NY, USA, 3rd edition, 1997.

[14] WHO, Training Course on Child Growth Assessment, World Health Organization, Geneva, Switzerland, 2008.

[15] WHO, Nutrition anaemia:Tools for Effective Prevention and Control, World Health Organization, Geneva, Switzerland, 2017.

[16] S. S. Alam, N. Rahman, A. Mia, M. Haque, and K. Islam, "Dietary diversity and nutritional status of adolescent girls in selected urban slum of dhaka city in Bangladesh," Nutrition \& Food Science International Journal, vol. 7, no. 3, pp. 1-5, 2018.

[17] S. Ahmad, P. Gupta, R. Khatoon, Z. H. Zaidi, and R. Sahai, "Prevalence of anaemia amongst adolescents attending OPD at rural health and training centre, Era's Lucknow Medical College and Hospital," International Journal of Community Medicine and Public Health, vol. 5, no. 9, pp. 4124-4127, 2018.
[18] M. Tesfaye, T. Yemane, and L. Gedefaw, "Anemia and iron deficiency among school adolescents: burden, severity, and determinant factors in southwest Ethiopia," Adolescent Health, Medicine and Therapeutics, vol. 6, pp. 189-196, 2015.

[19] Ghana National Anaemia Profile, 2014, http://www. springnutrition.org.

[20] S. S. Biradar, S. P. Biradar, A. C. Alatagi, A. S. Wantamutte, and P. R. Malur, "Prevalence of anemia among adolescent girls: a one year cross-sectional study," Journal of Clinical and Diagnostic Research, vol. 6, no. 3, pp. 372-377.

[21] A. Callister, J. Gautney, C. Aguilar, J. Chan, and D. Aguilar, "Effects of indegenous diet iron content and location on hemoglobin levels of Ghanaians," Nutrients, vol. 12, no. 9, p. $2710,2020$.

[22] L. Gosdin, A. Sharma, K. Tripp et al., "Barriers to and facilitators of iron and folic acid supplementation within a school-based integrated nutritiona and health promotion program among Ghanaian adolescent girls," Current Development in Nutrition, vol. 4, no. 9, pp. 1-11, 2020.

[23] S. D. Dubik, K. E. Amegah, A. Alhassan, L. N. Mornah, and L. Fiagbe, "Compliance with weekly iron and folic acid supplementation and its associated factors among adolescent girls in tamale metropolis of Ghana," Journal of Nutrition and Metabolism, vol. 2019, Article ID 8242896, 12 pages, 2019.

[24] P. Horjus, V. Aguayo, J. Roley, M. Pene, and S. Meershoek, "School-bades iron and folic acid supplementation for adolescent girls: findings from manica province,Mozambique," Food and Nutrition Bulletin, vol. 3, no. 26, pp. 281-286, 2005. 